\title{
Fabrication and Characterization of Semiconductor CuCl Nanocrystals
}

\author{
S. Mahtout, M.A. BelkhiR* and M. Samah \\ Solid physics group, University of Bejaia, 06000 Bejaia, Algeria
}

(Received August 7, 2003)

\begin{abstract}
$\mathrm{CuCl}$ nanocrystals were elaborated in a $\mathrm{NaCl}$ ionic matrix by doping the latter with copper powder during growth. Optical absorption measurements revealed nanocrystals with a mean size of order $32 \AA$. This is consolidated by the Raman scattering measurements which showed nanodomains of similar size. X-ray diffraction measurements indicate a good crystallinity of the matrix and confirm the presence of $\mathrm{CuCl}$ nanocrystals within our samples. The annealing effect at $300^{\circ} \mathrm{C}$ showed an increase in $\mathrm{CuCl}$ nanocrystal size with annealing time and demonstrated clearly the existence of a compound containing copper within our samples.
\end{abstract}

PACS numbers: 78.67.-n, 78.40.Fy, 78.40.-q

\section{Introduction}

Since the discovery of the transistor, there is more than fifty years, the semiconductors play a major role in electronics, and consequently in our everyday life. At the end of the seventies, with the introduction of new techniques of controlled crystal growth, such as metal-organic chemical vapour deposition or molecular beam epitaxy, etc., growth of solids of low dimensionality (two, one, or even zero) became possible. This type of materials is very attractive both for applications and theoretical investigations. Indeed, we may use them to fabricate lasers [1], optical amplifiers, or solar cells. In the field of electronics, the growing number of taken patents $[2,3]$ allows to suppose that the applications of nanocrystals will be very important in the near future. The work presented here consists in working out of $\mathrm{CuCl}$ nanocrystals in a $\mathrm{NaCl}$ ionic matrix and their characterization. Optical absorption measurements at ambient temperature are followed by a study

*corresponding author; e-mail: belkhir_a@yahoo.fr 
of the effect of thermal annealing on the optical absorption spectra. Then X-ray diffraction (XRD), photoluminescence, and Raman diffusion measurement are also studied.

\section{Experiment}

Our samples were fabricated using the Czochralski technique. The principle of the method consists in melting the material, which is to be crystallized, in a melting pot. Crystallization is then obtained due to a weak temperature gradient. The latter is settled by the formation of a meniscus around a seed crystal in contact with the melt surface. Doping of the melted $\mathrm{NaCl}$ was done at temperature averaging $900^{\circ} \mathrm{C}$. Chosen pulling parameters are one centimetre per hour and one turn per minute, respectively, for translation and rotation movements. One finally obtains pastilles in the form of discs with about $1 \mathrm{~mm}$ of thickness and $1 \mathrm{~cm}$ of diameter. These pastilles were then subjected to various methods of characterization.

\section{Results and discussion}

Optical absorption measurements of $\mathrm{NaCl}$ doped with copper were made on several samples. Figure 1 shows an example of the obtained spectra. The latter

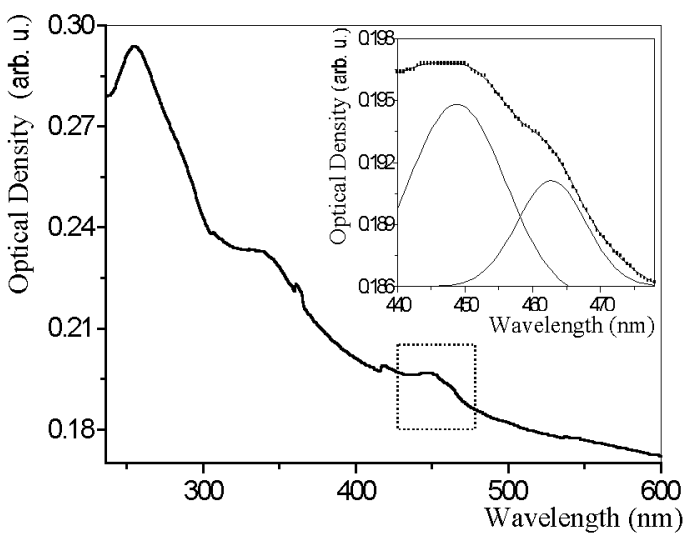

Fig. 1. Optical absorption spectrum of $\mathrm{NaCl}$ doped by copper.

presents several peaks in the visible and near ultraviolet. The peaks appear as a shouldering [4] (addition of peaks originated from different sizes). In the ideal case where the nanocrystals have all the same size, the peak should be very fine. The broadening of the peak tells us about the importance of the size dispersion of the nanocrystals [5]. The first observed peak is located at the neighbourhoods of $255 \mathrm{~nm}$. This one is allotted to the internal transitions from $\mathrm{Cu}^{+}$monomeric $[6,7]$. Another peak appears in the neighbourhoods of $340 \mathrm{~nm}$. At this stage, the nature 
of this peak is not known [8], but the effect of thermal annealing at $300^{\circ} \mathrm{C}$ shows a behaviour of a compound containing copper, as will be seen further. The very fine peaks which appear at 307,360 , and $417 \mathrm{~nm}$ in the spectrum are due to the change of the lamp of the measuring equipment. This change occurs automatically to sweep all the frequencies of the range of measurement. In the visible part of the absorption spectrum, one can see the existence of two peaks towards $435 \mathrm{~nm}$ and $469 \mathrm{~nm}$ shown in top of Fig. 1. According to Dijken et al. [9], these transitions are due to trap levels located in the forbidden band of $\mathrm{CuCl}$ nanocrystals. Figure 2 shows the part of Fig. 1 magnified in the region 368-378 nm. Fitting with four Gaussian functions, we obtain four lines located at positions: $368,371,373$, and $375.5 \mathrm{~nm}$. The first and the fourth are allotted to $Z_{1,2}$ and $Z_{3}$ excitons, respectively, with a spin-orbital splitting of $66 \mathrm{meV}$. This value is sensibly equal to that of $69 \mathrm{meV}$ given by Cardona [10] and Goldmann et al. [11]. The energy position of these excitons is shifted to the high energies compared to that of excitons in bulk crystal $[10,12]$. This is due to the quantum confinement effect. To estimate the size of nanocrystals, we used the formula of weak confinement given by Ekimov et al. $[13,14]$ :

$$
E=E_{\mathrm{g}}-E_{\mathrm{exc}}+\frac{\hbar^{2} \pi^{2}}{2 M a^{2}},
$$

where $E$ is the position of the excitonic line, $E_{\mathrm{g}}$ - the gap of the considered material, $E_{\text {exc }}$ - the binding energy of the exciton, $h$ - the Planck constant, $M$ - the total mass of the electron-hole quasiparticle, and $a$ - the nanocrystal radius assumed of spherical form. For $\mathrm{CuCl}$, we have $M=2.5 m_{0}, E_{\text {exc }}=152 \mathrm{meV}$ [15]. Knowing that the peak with $373.5 \mathrm{~nm}$ of Fig. 2 corresponds to that of $Z_{3}$ exciton, one finds a diameter $2 a=32 \AA$.

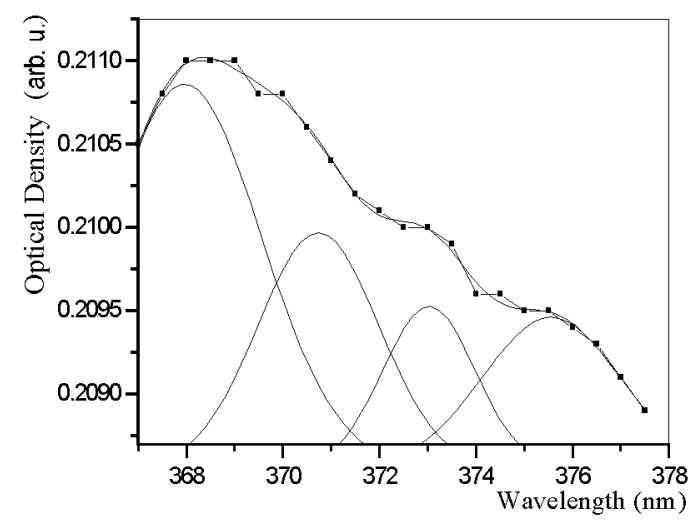

Fig. 2. Optical absorption spectrum of $\mathrm{CuCl}$ nanocrystals.

On the basis of the fact that energy bands, similar to those of excitons in bulk $\mathrm{CuCl}$, are observed in the transparent area of the absorption spectrum of 


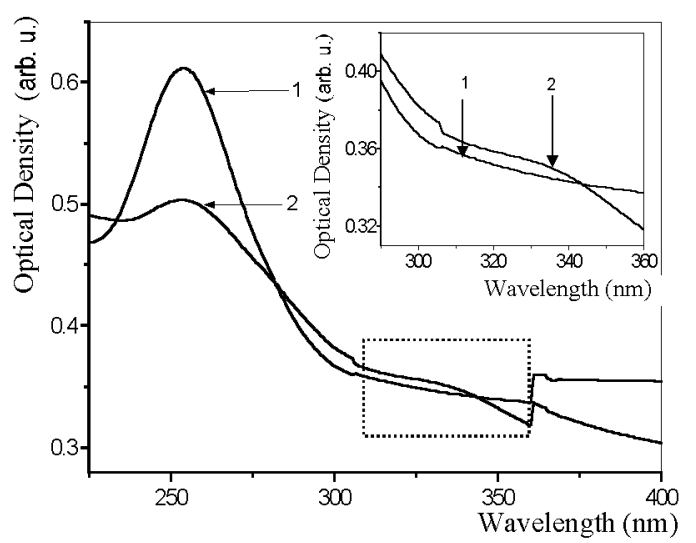

Fig. 3. Evolution of the optical absorption spectrum according to the annealing time at $300^{\circ} \mathrm{C}$ : (1) rough sample, (2) after 24 hours of annealing.

$\mathrm{NaCl}$, it is allowed to suppose the existence of nanocrystals of $\mathrm{CuCl}$ in a $\mathrm{NaCl}$ matrix strongly doped by copper. The origin of these bands is explained by the formation of nanocrystals of $\mathrm{CuCl}$ in the $\mathrm{NaCl}$ matrix by fixation of the $\mathrm{Cu}^{+}$ions. In this part, we will study experimentally the behaviour of the optical absorption spectra of the $\mathrm{NaCl}$ samples doped by copper, according to the annealing time at $300^{\circ} \mathrm{C}$. Optical absorption measurements are taken after each stage of annealing. The results are presented in Fig. 3 for rough sample (curve 1) and after 24 hours of annealing (curve 2 ). The remaining results are given in Table. During the

TABLE

Evolution according to time of the $\mathrm{CCC}$ peak with respect to the $\mathrm{Cu}$ peak at $300^{\circ} \mathrm{C}$.

\begin{tabular}{c|c|c}
\hline \hline Time [h] & Peak Cu [arb.u.] & Peak CCC/Peak Cu \\
\hline 0 & 0.6124 & 0.5452 \\
8 & 0.5884 & 0.5781 \\
12 & 0.5724 & 0.6163 \\
16 & 0.5469 & 0.6328 \\
24 & 0.5039 & 0.6945
\end{tabular}

various stages of annealing, we note, according to time, a progressive reduction of the line corresponding to the $\mathrm{Cu}^{+}$ions (to $255 \mathrm{~nm}$ ) and a simultaneous and progressive appearance of the line at $340 \mathrm{~nm}$ as shown in the top of Fig. 3. It is on the basis of this particular behaviour that we allot this peak to a compound containing copper (CCC). This result can be explained by the fact that because of annealing at $300^{\circ} \mathrm{C}$, the aggregates of copper ions, inside the sample, are dispersed in the form of separated ions and their interaction with the matrix and with the 
other elements which might exist as impurities gives rise to aggregates of this material. The increase in the peak corresponding to the CCC can be explained by the increase in the number of aggregates of this material. The optical absorption spectrum of $\mathrm{CuCl}$ nanocrystals, after 24 hours of annealing is shown in Fig. 4. We observe a displacement of the $Z_{3}$ peak position of $375 \mathrm{~nm}$ at $379 \mathrm{~nm}$ (towards weak energies). The shift of the $Z_{3}$ exciton peak toward the long wavelength side is attributed to the increase in the size of $\mathrm{CuCl}$ nanocrystals with annealing time. This displacement induces an increase in the $\mathrm{CuCl}$ nanocrystal size of $32 \AA$ to $50 \AA$. This behaviour is explained by the quantum confinement effect; when the size increases, the quantum confinement effect decreases and the energetic position of $\mathrm{CuCl}$ excitons passes towards weak energies.

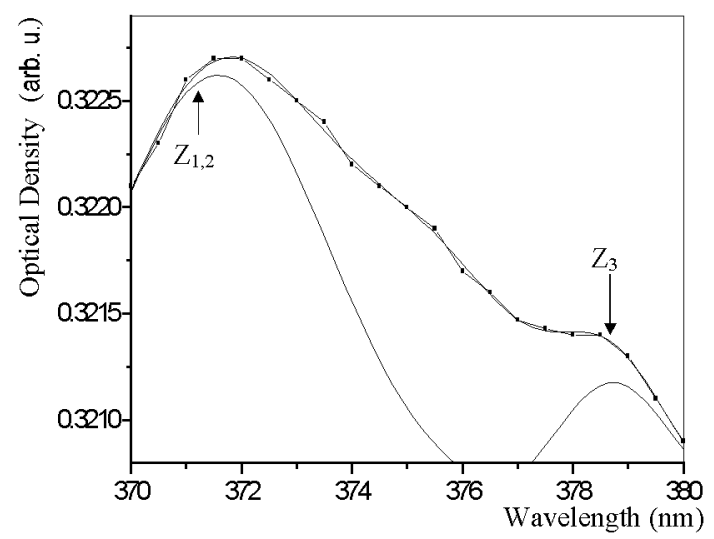

Fig. 4. Optical absorption spectrum of $\mathrm{CuCl}$ nanocrystals after 24 hours of annealing at $300^{\circ} \mathrm{C}$.

XRD measurements were taken on several samples and the obtained spectra are all identical. Let us note that, by using a monochromator, only the line $K_{\alpha}$ of copper was selected from the source of X-rays. The spectrum of Fig. 5 presents an example of these results. This one consists of two principal lines with the positions $2 \theta=31.68^{\circ}$ and $2 \theta=66.24^{\circ}$. The first corresponds to the diffraction from the plane (200) of $\mathrm{NaCl}$ and the second to its harmonic (400). This rises from the comparison of the spectra obtained according to ASTM card (American Society for Testing and Materials) of $\mathrm{NaCl}$. The appearance of these two harmonics confirms well the good monocrystallinity of the fabricated matrix. The other lines, of low intensity compared to those of the matrix, appear at $2 \theta=28.39^{\circ}, 2 \theta=$ $33.32^{\circ}, 2 \theta=47.52^{\circ}, 2 \theta=56.32^{\circ}, 2 \theta=69.44^{\circ}$, and $2 \theta=76.68^{\circ}$. The latter correspond, according to ASTM cards, to the plane (111), (200), (220), (311), (400), and (331), respectively, of a $\mathrm{CuCl}$ crystal of zinc blende structure with a cell parameter of $5.416 \AA$. This result confirms the presence of $\mathrm{CuCl}$ nanocrystals in the matrix. 


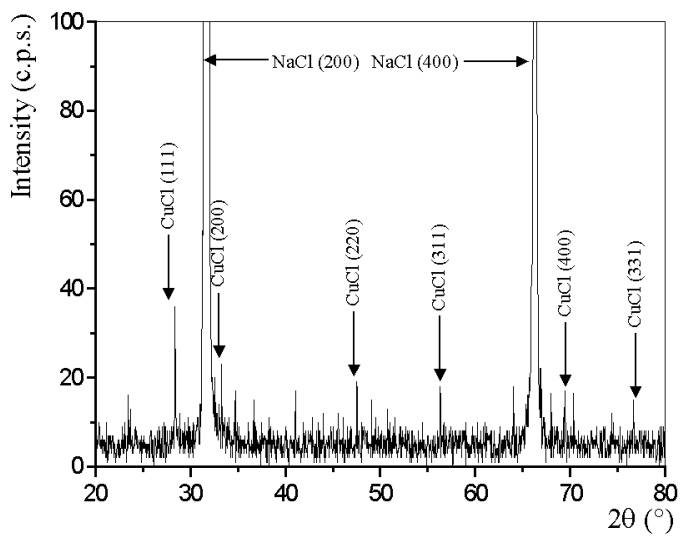

Fig. 5. XRD spectrum of $\mathrm{NaCl}$ sample doped by copper.

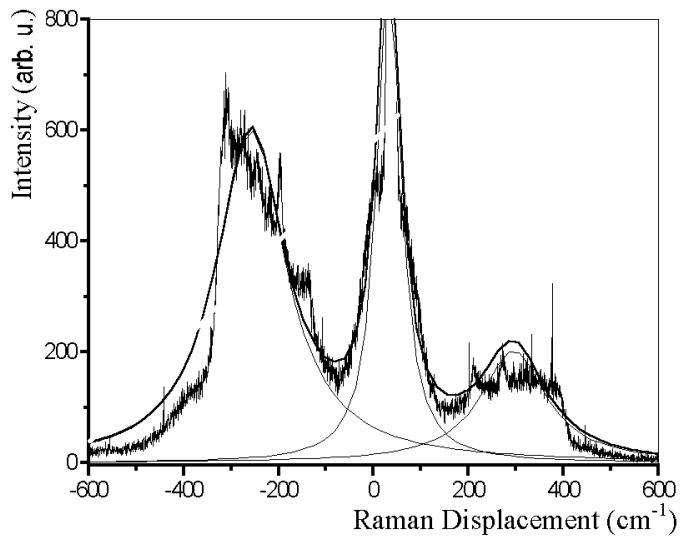

Fig. 6. Raman diffusion spectrum of $\mathrm{NaCl}$ sample doped by copper.

The results of measurement of the Raman diffusion on the $\mathrm{NaCl}$ sample doped by copper are presented in Fig. 6. The Raman diffusion low frequency can reveal the presence of nano-heterogeneities inside our samples [16, 17]. The presence of the band at very low frequency $37 \mathrm{~cm}^{-1}$ was connected with the inelastic scattering of the light by the natural vibrations of nanodomains in the $\mathrm{NaCl}$ matrix. As one can see it on the same figure, we have in addition a Stokes band at $-280 \mathrm{~cm}^{-1}$ and an anti-Stokes band at $+280 \mathrm{~cm}^{-1}$ in relation to these nanodomains.

The size of these nano-heterogeneities is estimated by the formula of Duval et al. [16]:

$$
2 a=\frac{s v}{c \omega}
$$

where $v$ is the sound velocity in the nanodomain, $c$ - the celerity of the light in 
the vacuum, $2 a$ - the diameter of the particle, $\omega$ - the position of the Raman band low frequency, and $s$ - the form factor which varies approximately between 0.5 and 0.8 according to the shape of the nanodomain. In our case where the sound velocity is on a verage equal to approximately $5300 \mathrm{~m} / \mathrm{s}$ and where the band position is $\omega=37 \mathrm{~cm}^{-1}$, one finds a nanodomain average size of $2 a=31 \AA$. In good agreement with the value of the average size estimated by the optical absorption measurements, this result confirms well that this nanodomain represents the $\mathrm{CuCl}$ nanocrystals.

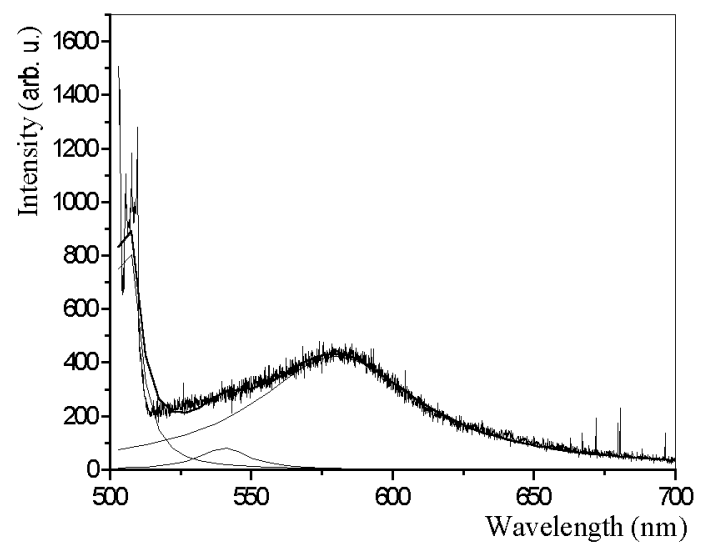

Fig. 7. Photoluminescence spectrum of $\mathrm{NaCl}$ sample doped by copper.

Measurements of photoluminescence were taken at the ambient temperature with an excitation of $488 \mathrm{~nm}$ which appears in the obtained spectrum (Fig. 7) at the same position. This spectrum shows a very apparent peak at $576 \mathrm{~nm}$ very broadened on the side of low wavelengths. The deconvolution of the curve by two Gaussians shows the existence of two peaks at $539 \mathrm{~nm}$ and $579.5 \mathrm{~nm}$. These are allotted to the emission of defect such as the F-centre in the samples [18], explaining the greenish-yellow colour of the samples. Indeed, the wavelength $539 \mathrm{~nm}$ corresponds to the green and $579.5 \mathrm{~nm}$ to the yellow. The peaks corresponding to $\mathrm{CuCl}$ are not observed, since the energy of the radiation used $(2.54 \mathrm{eV})$ is not sufficient to excite the levels of $Z_{1,2}$ and $Z_{3}$ excitons which are located between $3.2 \mathrm{eV}$ and $3.4 \mathrm{eV}$ ( 387.5 and $364.5 \mathrm{~nm}$ ).

\section{Conclusion}

In this work we succeeded to fabricate the $\mathrm{CuCl}$ nanocrystals by doping the $\mathrm{NaCl}$ matrix during growth by a fine powder of copper. The size of $\mathrm{CuCl}$ nanocrystals is estimated to be $32 \AA$. The displacement of the excitonic peak position of $\mathrm{CuCl}$ towards high energies is due to the quantum confinement effect. 
$\mathrm{XRD}$ and Raman scattering measurements confirmed well the formation of $\mathrm{CuCl}$ nanocrystals of zinc blende structure and a cell parameter of $5.14 \AA$. The study of the thermal annealing effect at $300^{\circ} \mathrm{C}$ showed an increase in the size of $\mathrm{CuCl}$ nanocrystals with annealing time and shows that the peak which appears in optical absorption measurements at $340 \mathrm{~nm}$ belongs to a compound containing copper.

\section{References}

[1] R.W. Siegel, E.Hu, D.M. Kox, H. Goronkin, L. Jelinski, C.C. Koch, J. Mendel, R.C. Roco, D.T. Chaw, WTEC US Pannel Rapport International Technology Research Institut, 1999.

[2] P. Alivisatos, V. Colvin, U.S. Patent 5 018, 751, 1989.

[3] M. Bawendi, K. Jensen, B. Daboussi, J. Rodrigguez-Viego, F.V. Mikulec, U.S. Patent 6, 207, 2001.

[4] M.A. Chamarro, V. Voliotis, R. Grousson, P. Lavallard, T. Gacoin, G. Counio, J.P. Boilot, R. Cases, J. Cryst. Growth 159, 853 (1996).

[5] O. Lublinskaya, S. Gurevich, A. Ekimov, I. Kudryavtsev, A. Osinskii, M. Gandais, Y. Wang, J. Cryst. Growth 158, 203 (1995).

[6] H. Kishishita, Phys. Status Solidi B 55, 399 (1973).

[7] S. Nagasaka, M. Ikezawa, M. Veta, J. Phys. Soc. Japan 20, 1450 (1995).

[8] T. Itoh, Phys. Status Solidi B 145, 567 (1988).

[9] A. Van Dijken, E.A. Meulenkamp, D. Vanmaekelbergh, A. Meijerink, J. Lumin. 90, $123(2000)$.

[10] M. Cardona, Phys. Rev. 129, 69 (1963).

[11] A. Goldmann, J. Tejeda, N.J. Shevchik, M. Cardona, Solid State Commun. 15, 1093 (1974).

[12] S. Park, I. Kim, K. Jang, S. Kim, C.D. Kim, Y. Yee, G. Jeen, J. Phys. Soc. Jpn. 70, 3723 (2001).

[13] A.I. Ekimov, Al.L. Efros, A.A. Onushchenko, Solid State Commun. 56, 921 (1985).

[14] A.I. Ekimov, A.A. Onushchenko, A.G. Plyukhin, Al.L. Efros, Sov. Phys.-JETP 61, 891 (1985).

[15] P. Gilliot, Ph.D. thesis, Université de Strasbourg, France, 1990.

[16] E. Duval, A. Boukenter, B. Champagnon, Phys. Rev. Lett. 56, 2052 (1986).

[17] M. Philippe, Phys. Rev. Lett. B 77, 557 (1997).

[18] C. Kittel, Solid State Physics, 5th ed., Dunod, Paris 1983 (in French). 